\title{
A new method for time calibration of substation automation equipment based on synchronous closed loop monitoring
}

\author{
YANG Sheng \\ (NARI Technology Co.LTD., Jiangsu Nanjing, 211106, China) \\ yangsheng@sgepri.sgcc.com.cn
}

Keywords: Time synchronization; Closed loop check; Time information flow; Intelligent substation.

\begin{abstract}
The on-line monitoring of time synchronization system in Smart Substation is the key point to solve the time traceability of smart substation. Analyze current status of time synchronization in substation time synchronization system. Point out the defects of low time precision, abnormal synchronization and time service of microsecond equipment. Propose a method of synchronous closed-loop monitoring of substation automation equipment calibration method based on time, by the state monitoring module, connection time, send port part time information part, return time status information, time deviation calculation part, determine the abnormal and error and time error correcting part is composed of seven parts. Analyze the principle and implementation method. Propose the time information flow for actual substation. Through close loop check, the time condition of substation automation equipment can be accurately detected, and solve the clock synchronization problem of intelligent substation.
\end{abstract}

\section{Introduction}

With the rapid development of the the smart grid automation level, the problems of the time system of the substation automation equipment are more prominent.

All kinds of automation devices based on computer and communication technology are widely applied in smart grid.

In the current smart grid, the timing between the clock device and the timing device adopts the open loop mode, which lacks effective detection measures for the clock synchronization state and timing accuracy of various devices.

When the fault occurs, the accident analysis finds that the time of the protection and the backstage events in the station and the station are inconsistent or even contradictory. It affects the overall, objective and accurate analysis of the accident, and also affects the accuracy of the analysis results.

To solve the above problems and the present situation, this paper puts forward a kind of intelligent substation synchronization verification method based on synchronous closed-loop monitoring, on-line monitoring system for the closed-loop system of intelligent substation time synchronization system solves the problems caused by the defects on the system, provides a reasonable solution.

\section{Traditional time system and defects}

The hierarchical management mode is used on intelligent substation system, the station control layer host through SNTP protocol message management spacer device based on time, and the control device spacer through message management process GOOSE protocol layer based devices, the main mode of the following defects:

1) the low precision background host is the arbiter. In general sense, the time accuracy of the background host is second level. It is one of the lowest accuracy devices in the two substation of the whole substation. It is unreasonable to arbitrate other high-precision devices with the backstage host.

2) there may be time service in the background itself. As a timing device, the background host itself has the possibility of synchronous abnormality. Because there is no device monitoring the 
synchronization state of the background host, there is uncertainty in the background host itself monitoring.

3 ) it is unable to meet the monitoring requirements of microsecond time service equipment. The current time synchronization online monitoring method is a simple network monitoring method based on NTP and GOOSE. The accuracy is only millisecond, and it can't monitor the microsecond level of PMU and merging units.

\section{Time check method based on synchronous closed loop monitoring}

In view of the following problems, a solution based on synchronous close loop monitoring is proposed.

1) how to accurately detect the working conditions of the current station automation equipment;

2) how to form a closed loop monitoring between the time equipment and the time equipment, instead of monitoring and correcting the time precision through other third party equipment.

3 ) how to realize the precision monitoring and correction of the equipment by the time equipment.

In order to achieve the above purpose, this paper will synchronize the 7 modules of the close - loop - time - check division, and adopt the following scheme:Synchronous closed-loop monitoring of new substation automation equipment for calibration method based on condition monitoring module, connection port, send information on part time, return time status information, time deviation calculation part, determine the abnormal and error and time error correcting part part;

In the part of the state monitoring module, the time state monitoring module is added to the time equipment of the substation.

The time port is connected to the time port, which will be connected to the time port by the time device through the optical fiber or other communication medium.

Sending the time information part, the timing device sends the time information to the times text at the specified interval period,

The return time information part, sents to the timing device by the time interval information of the time equipment according to the time interval.

The time offset value is calculated. The time difference between the time and standard time of the two time is calculated for the time equipment which is compared with the timing information returned by the time equipment.

The anomaly and the error part are determined, and the time state anomaly and the time error of the equipment at the moment are determined according to the time precision rule.

The time error is corrected by the time error, and the time error of the equipment is corrected by the time error according to the time error.

\section{Implementation of synchronous close loop monitoring system for time check}

Time synchronization management module synchronization equipment, as the station station level time synchronization monitoring and management, realize the principle of substation in SNTP table tennis all the typical based devices, such as clock device, protection device, control device, communication device, fault recorder, time synchronization monitoring; measurement and control device as the spacer layer management, the realization of smart terminal, merging unit, learningage equipment integrated clock synchronization monitoring.

The clock device, the state information of the timing device, as shown in Table 1, table 2. 
Table 1 clock device status information

\begin{tabular}{|l|l|}
\hline \multicolumn{2}{|c|}{ Table 1 clock device status information } \\
\hline Description & Definition \\
\hline antenna state & normal, abnormal, malfunction \\
\hline Satellite module state & $\begin{array}{l}\text { normal, abnormal, abnormal self } \\
\text { inspection }\end{array}$ \\
\hline Power module state & Normal, power module exception \\
\hline Clock hopping detection state & Normal, abnormal, detects time jump \\
\hline Time source selection & Current time source \\
\hline Initialization stat & Electrical reset on the equipment \\
\hline Crystal tamed state & Normal, crystal tamed abnormality \\
\hline
\end{tabular}

Table 2 The state information table of the time given equipment

\begin{tabular}{|l|l|}
\hline Description & Definition \\
\hline Time signal state & $\begin{array}{l}\text { Normal, abnormal, error checking, } \\
\text { ineffective quality, no signal }\end{array}$ \\
\hline Time service state & Normal, abnormal, equipment no timing \\
\hline Time jump detection state & Normal, abnormal, detects time jump \\
\hline
\end{tabular}

A new type of time check system for substation automation equipment based on synchronous closed loop monitoring, the structure diagram is shown as follows:

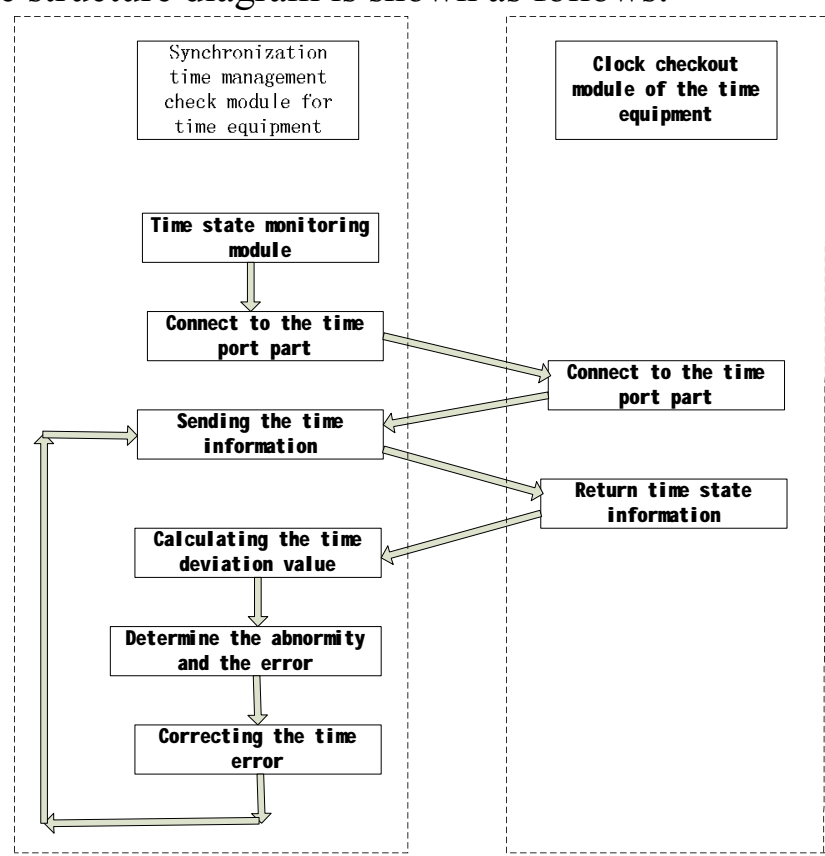

Fig. 1 structure and working flow chart of synchronous closed loop time check system The working process is as follows:

Step 1: the time state monitoring module of the clock device starts and starts to synchronize the closed loop checkout process.

Step 2: the connection of the clock device is started on the time port, and the time port is connected by the optical fiber or other communication medium.

Step 3: the connection to the time port is started by the connection of the timing device, and the time port is connected by the optical fiber or other communication medium to the time port.

Pairs of channels;

Step 4: the time information part of the clock device is sent, and the time message is sent to the times by the prescribed interval period.

Step 5: the time information portion is started by the return of the timing device, and the timing device is returned to the clock device by the time interval of the timing device for the prescribed interval.

Step 6: clock timing of the clock device starts, and the time difference between the time information and the standard clock returned by the time device is compared to the time equipment, and the deviation between the two time and the standard time is calculated. 
Step 7: clock device's decision abnormality and error part start. According to the rule of timing accuracy, the timing state anomaly and time error of the time service device are determined.

Step 8: the timing error of clock device is partly activated, and the timing equipment based on the above time error is corrected in the next time, and the time error of equipment is corrected.

\section{Synchronous close loop monitoring of time check system against time data flow}

The time synchronization state information flow of all devices in a typical substation is composed of the sending port, the receiving terminal, the communication protocol and the content of information.

The clock time information transmission equipment for the station source, through the station control layer network to the station is on state measurement data transmission timing of the device, according to different equipment, can use NTP/IEC61850/IEC103/IEC104 protocol; all were sent to the clock synchronization and timing device state measurement data, self and self checking equipment status data, can be used the original station control layer protocol IEC61850/IEC104/DL476/.

\section{Conclusions}

In this paper, a new method of time check for a new type of substation automation equipment based on synchronous closed loop monitoring is proposed. Through the synchronous closed loop check, it can accurately detect the time condition of the substation automation equipment. Close loop monitoring is formed between the time equipment and the equipment that is on time, instead of monitoring and correcting the time accuracy through other third party equipment. The precision monitoring and correction of the equipment are realized by the synchronous closed loop. It is simple in principle, high in reliability, high in accuracy and convenient in use. It has very high practical value of engineering, and it has solved the problem of the time system of the intelligent station.

\section{References}

[1] S. Miao, S. Wang. The Design and Implementation of the GPS based Real-time Monitoring System for the State of Power Network[J].International Journals of Power \& Energy Systems,2001,21(3):159-163

[2] Holbert. K.E., Heydt. G.I, Ni, H. Use of Satellite Technologies for Power System Measurements, Command, and Control[J].Proceedings of the IEEE, 2005,93(5):947-955

[3] Holbert. K.E., Heydt. G.I, Ni, H. Use of Satellite Technologies for Power System Measurements, Command, and Control[J].Proceedings of the IEEE, 2005,93(5):947-955

[4] E. Y. Song, K. Lee. An Application Framework for the IEEE 1588 Standard. Proceedings of the 2008 IEEE International Symposium on Precision Clock Synchronization(ISPCS) for Measurement, Control and Communication, Ann Arbor, 2008:2 3 